\title{
Efficacy of Stent Implantation Combined with Balloon Dilatation in the Treatment of Severe Peripheral Arterial Occlusive Disease of Lower Extremity in Macao-A Single Center Experience
}

\author{
Ieong Chon Man $^{1 *}$, Liang Gang Zhu ${ }^{2}$, Tam Man Pan ${ }^{1}$, Kong Soi Chau ${ }^{1}$, Kuok Cheong ${ }^{1}$ \\ ${ }^{1}$ Department of radiology, Conde de São Januário Hospital, Macao, China \\ ${ }^{2}$ Department of vascular surgery, Conde de São Januário Hospital, Macao, China \\ *Email: benjaminicm@yahoo.com.hk
}

\begin{abstract}
Objective: To investigate the feasibility and clinical efficacy of stent implantation and balloon angioplasty in the treatment of severe lower extremity arterial ischemia. Methods: Twentytwo patients with severe lower extremity ischemia were enrolled from January 2016 to December 2016. According to the scope of the patient's lesions, we choose the appropriate length of the balloon, according to different degrees of balloon dilation lesion stenosis or occlusion of the adjacent multiple short part of the lesion, as far as possible the formation of long balloon. Metallic stents were implanted in the dilated vessels to prevent restenosis. The incidence of postoperative complications, the rate of technical success and the clinical efficacy were observed, and the primary patency rate was evaluated through follow-up. Results: The total of 22 patients had 42 vessels with varying degrees of stenosis and occlusion, with an operative success rate of $100 \%$. The primary patency rate was $90.9 \%$ and $81.8 \%$ at 6 and 12 months respectively. The ankle brachial index was significantly higher than that before the operation, and the Rutherford classification of most patients was significantly improved after the operation $(\mathrm{P}<0.05)$. No serious complications or death cases were found in this study. The average postoperative hospital stay was 4.3 days. Conclusion: For the severe ischemic disease of the lower extremities, interventional therapy is more effective than surgical treatment, with fewer complications and faster recovery. Stent implantation combined with balloon therapy does not affect the postoperative blood supply of the affected limb. The initial technical and clinical success rate is very high. It is a safe and effective method for the treatment of severe arterial ischemia disease of the lower extremities.
\end{abstract}

Keywords: Peripheral arterial occlusive disease, stent implantation, balloon dilatation, treatment

\section{Introduction}

Peripheral arterial occlusive disease can cause peripheral artery ischemia, leading to local pain and necrosis, ulcers, infection, and even amputation. The current treatment methods include angioplasty, endarterectomy, balloon angioplasty and stent implantation, and feasible bypass grafting. [1-2] Peripheral arterial occlusive disease is not uncommon in clinical practice. With the improvement of people's living standard, life expectancy, living habits and metabolic diseases, the incidence of this disease is increasing. The peripheral arterial occlusive disease often leads to severe limb ischemia, which causes the risk of amputation, and requires early revascularization. At present, interventional therapy has gradually become one of the better treatments for this disease. From January 2016 to December 2016, 22 cases of lower extremity arterial occlusive disease were treated in our hospital. All of them were treated with intravascular treatment and the effect was good.

\section{Methods}

Patients: All patients received informed consent prior to any operation and the study was approved by the ethics committee of our hospital. This retrospective study of endovascular treatment of PAOD from January 2016 to December 2016 was performed.

Procedural technique: All patients underwent preoperative vascular ultrasound examination, CTA 
confirmed lower limb lesions. The location, extent, collateral circulation and outflow tract of the lesion were confirmed. The ankle brachial index (ABI) was compared before and after operation in all patients. All surgical methods of each patient were based on CTA results to determine the degree of stenosis and choose surgical approach. The femoral artery puncture was performed by the same side antegrade puncture or contralateral retrograde puncture, and the puncture was performed under the guidance of ultrasound. On the basis of understanding the patency of femoral artery, ipsilateral femoral artery puncture was performed. Then, (activated clotting time) ACT was examined during the operation, and then heparin was administered to the whole body (first dose of heparin $80 \mathrm{U} / \mathrm{kg}$, followed by $18 \mathrm{U} / \mathrm{kg}$ per hour). Selecting the appropriate catheter wire system and under the guidance of fluoroscopy, the closed segment of the artery occlusion was slowly opened. For patients with severe vascular calcification, 0.018 to 0.035 hard ultra-smooth guidewire is used to open the occluded artery. After the guidewire passes through the stenosis or occlusion segment, the stenosis or occlusion segment is expanded by using a balloon with different diameter $(2-8 \mathrm{~mm})$ and length $(4-12 \mathrm{~cm})$ (Boston Scientific, USA), while the adjacent multiple short stenoses are used as long as possible by using a long balloon. In the dilatation process, we should also appropriately expand the pressure of balloon dilation according to the patient's tolerance to pain. Each balloon dilation time was about 1-3 minutes. Stents were implanted in the diseased vessels after balloon dilatation (Supera, Abbott, USA or Innova, Boston Scientific, USA ). If the arterial lesions below the knee should be treated once, avoid repeated expansion and lead to arterial spasm.

Follow up: All cases were given (ankle brachial pressure index) ABI measurements at 1 days after the operation. The patients were followed up for 1 months and every 3 months after discharge.

In order to prevent postoperative arterial occlusion again, we give the patients with clopidogrel $75 \mathrm{mg}$ orally every day after operation for 1 months and Aspirin Enteric-coated Tablets 100mg day, long-term use CTA examination would be performed every 3 months post operation to assess the condition of recanalization and restenosis.

Endpoints: The clinical symptoms, signs and ABI changes of the patients before and after intervention were observed. The patency is defined as (1) Patency is defined as: there is no significant reduction in ABI (0.2 or more). (2) There was no significant restenosis (50\% or more) in the involved vessels, which was confirmed by CTA examination after operation. The success of the operation was defined as the status after the stent implantation, and the degree of residual lumen stenosis determined by immediate postoperative angiography was less than $30 \%$. The definition of clinical success is to increase the least one level by the RUTHERFORD grading system after 3 months of the operation.

Data analysis: SPSS version 21 is used as data analysis. The ABI values before and after the operation were analyzed by t test. The Rutherford grade scores before and after the operation were analyzed by the Wilcoxon rank sum test.

Table 1. Patient characteristics that underwent endovascular intervention for PAOD.

\begin{tabular}{ll}
\hline Patient characteristics & No. $(\%)(\mathrm{N}=22)$ \\
\hline Age, mean, years & $71.18+9.37$ \\
Gender & \\
Male & $18(81.8 \%)$ \\
Female & $4(18.2 \%)$ \\
Diabetes & $12(54.5 \%)$ \\
Current smoker & $14(63.6 \%)$ \\
Hypertension & $20(91.2 \%)$ \\
Coronary heart disease & $19(86.4 \%)$ \\
Hyperlipidemia & $11(50 \%)$ \\
PAD & $22(100 \%)$ \\
Preoperative medication & \\
Aspirin & $18(81.8 \%)$ \\
Clopideogrel & $20(91.2 \%)$ \\
Anti-coagulopathy medication & $10(45.5 \%)$ \\
Statin & $20(91.2 \%)$ \\
ABI index & $0.32 \pm 0.11$ \\
\hline
\end{tabular}


Table 2. Lesion characteristics.

\begin{tabular}{ll}
\hline Procedural variables & $\mathrm{No}(\%(\mathrm{~N}=22)$ \\
\hline Rutherford classification & \\
Grade 0 & 0 \\
Grade 1 & 0 \\
Grade 2 & 0 \\
Grade 3 & $12(54.5 \%)$ \\
Grade 4 & $4(18.2 \%)$ \\
Grade 5 & $6(27.3 \%)$ \\
Grade 6 & 0 \\
TASC II classification & \\
A & $5(22.7 \%)$ \\
B & $5(22.7 \%)$ \\
C & $6(27.3 \%)$ \\
D & $7(27.3 \%)$ \\
Involved arteries & \\
Common iliac artery & $5(11.9 \%)$ \\
Superficial femoral artery & $16(38.1 \%)$ \\
Popliteal artery & $5(11.9 \%)$ \\
Femoral profunda artery & $2(4.8 \%)$ \\
Anterior tibial artery & $6(14.3 \%)$ \\
Peroneal artery & $5(11.9 \%)$ \\
Posterior tibial artery & $3(7.1 \%)$ \\
Total & 42 \\
Length of occlusion, cm & \\
Mean \pm SD & $22.5 \pm 6.3$ \\
Range & $5-35$ \\
\hline
\end{tabular}

\section{$3 \quad$ Result}

\section{Patient characteristics}

The mean age of the 22 patients was $71.18+9.37$ years (53 84 years). Gender: male 18 cases, female 4 cases. We graded the patients by Rutherford classification. After assessment, there were 12 cases (54.5\%) in Rutherford grade III, 4 cases in grade IV (18.2\%), 6 cases in grade V (27.4\%), including 4 cases of toe ulcer and 2 cases of heel ulcer. These patients have different chronic diseases. The rates of coronary heart disease, hypertension, diabetes and hyperlipidemia were $88.5 \%, 91.2 \%, 59.1 \%$ and $50 \%$, respectively (Table 1). 13 patients suffered from varying degrees of renal insufficiency. Before the preoperative glomerular filtration rate was measured, the patients were given intravenous infusion during the perioperative period, and the amount of contrast media was reduced as much as possible. The glomerular filtration rate was assessed by the patients with poor renal function after operation.

\section{Lesion characteristics}

According to the results of CTA examination, 42 vessels were involved in our cases. Among them, the superficial femoral artery accounted for 16 (38.1\%), common iliac artery, deep femoral artery, popliteal artery, anterior tibial artery, posterior tibial artery and the peroneal artery accounted for $5(11.9 \%)$, $2(4.8 \%), 5(11.9 \%), 6(14.3 \%), 3(7.1 \%), 5(11.9 \%)$ cases respectively. The average length of obstruction or stenosis of the affected vessels was $22.5 \pm 6.3 \mathrm{~cm}$ (See table 2 ).

\section{Therapeutic effect}

All patients were successfully treated with angioplasty and stent implantation, and the operative success rate was $100 \%$.

In the evaluation of the curative effect, we rated the patients with the RUTHERFORD scoring system before the operation. We rated the patients before the operation and 1,3,6,12 months after the operation and found a significant improvement in the RUTHEFORD score in all patients $(\mathrm{P}<0.05)$. The postoperative clinical success rate was $95 \%$. 




Figure 1. Evolution of the Rutherford Category.

All patients were rated with Rutherford classification before the operation and at 1, 3, 6, and 12 months after the operation. The results showed that the patients were improved after the operation. After analysis, the results were $\mathrm{p}<0.05$. It shows that the two are statistically significant.

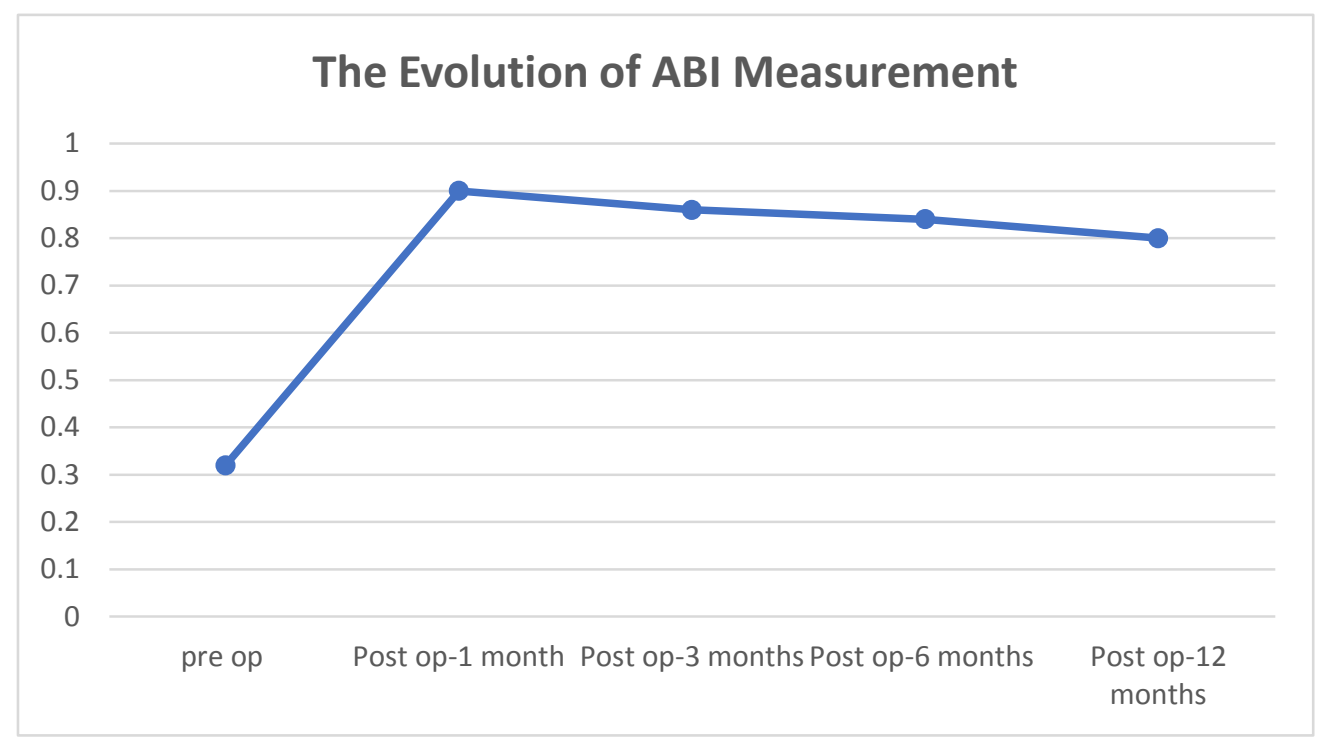

Figure 2. The change of ABI index.

From the change of ABI, we could see that ABI increased significantly in 1 month after operation, although the ABI index dropped slightly after 12 months, but the overall improvement was obvious. And it's statistically significant $(\mathrm{P}<0.05)$. 


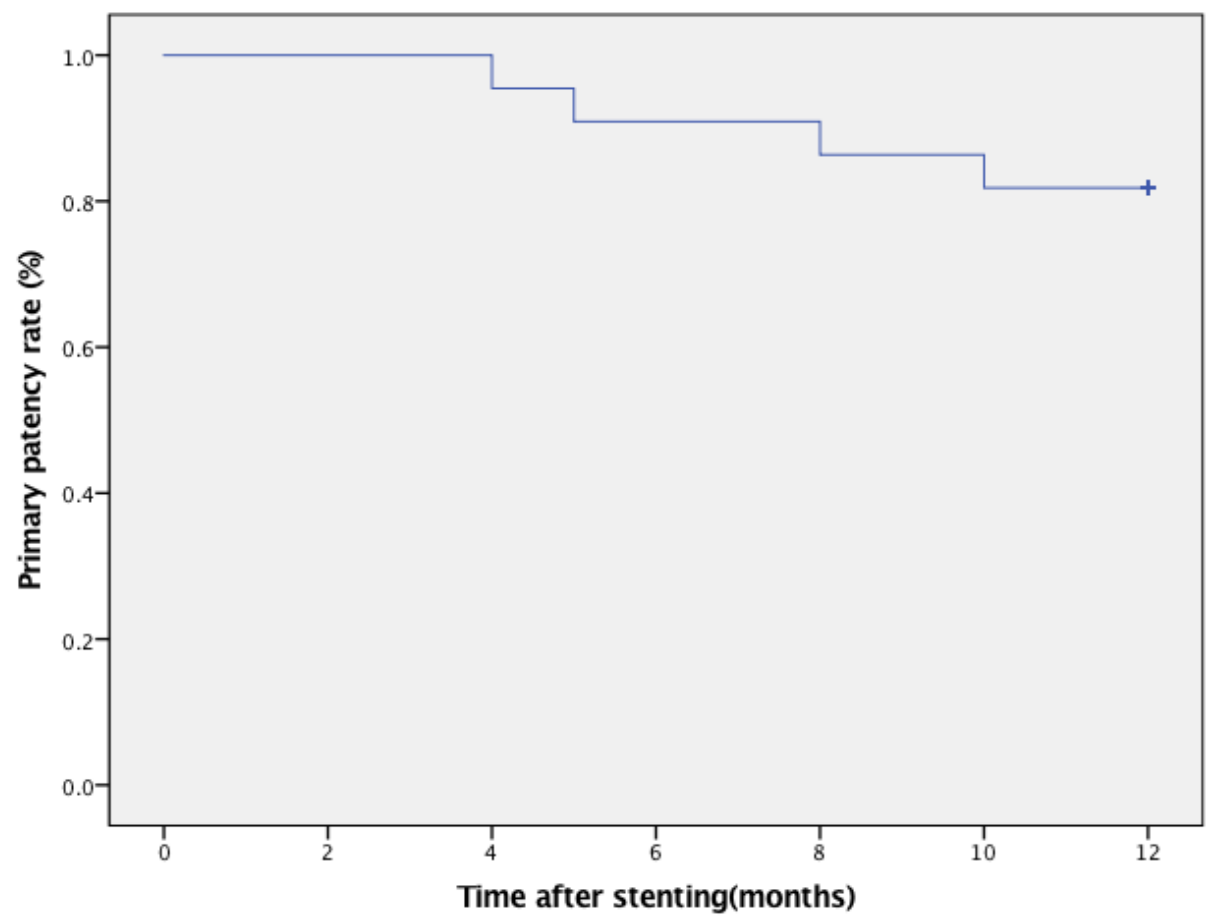

Figure 3. Primary patency after stenting.

The primary patency rate was $90.9 \%$ and $81.8 \%$ at 6 and 12 months respectively.

\section{Discussion}

With the aging of the population, patients with Chronic limb ischemia(CLI) have brought pressure to the treatment because of the complexity and complication of the disease. In 2015, a major global study found that about 155 million people around the world had PAD. [4] Therefore, patients with PAOD must be treated as early as possible to restore the blood supply of the distal extremities. For the treatment of CLI, traditional bypass is the main method for the treatment of CLI lesions, but there are many disadvantages, such as large trauma and many complications. And the long-term effect of bypass surgery on the below knee artery is not ideal. [5] Endovascular treatment has gradually become the main method for the treatment of PAOD. In the past, angioplasty was mainly used for balloon dilatation. But the incidence of restenosis is high. Baerlocher published a META analysis of standard balloon dilatation in PAOD. [6] It is suggested that the rate of restenosis is high after standard balloon dilatation. Therefore, stent implantation is becoming a major technique for the treatment of PAOD intravascular endovascular treatment. A META analysis of the long-term effects of balloon dilatation after balloon dilation was published by the Bosch et al. The long-term effect of balloon dilatation combined with stenting on PAOD is superior to that of balloon dilatation [7].

George et al. published an article this year, using the same type of scaffolding as this one. Their results showed that the primary patency rate was $96.9 \%$ at 6 months and $85.8 \%$ at 12 months. In our study, the primary patency rate was $90.9 \%$ and $81.8 \%$ at 6 and 12 months respectively, which was in accordance with the results of the study of George et al. (see figure 3).

This group of patients after interventional therapy, skin temperature, lower extremities blood supply was obviously improved, Dorsalis pedis artery pulse, limb pain and claudication were improved. The clinical symptoms, signs and the difference of ABI before and after intervention were statistically significant. The results of our study are consistent with the results of other foreign literature. [9-15] And no patient has serious complications such as myocardial infarction, heart failure, renal failure and so on, and the clinical effect is obvious. Although the recent effect of the interventional treatment of PAD is good, there are still many difficulties, and the effect is still to be further studied. The long-term and short-term patency rate of interventional treatment for below the knee artery is inferior to that of above the knee artery. The main 
reason is that the diameter of artery is thin, blood flow is slower, and temporary arterial blood flow is temporarily interrupted in balloon dilation. The main reason is that the cumulative interruption of blood flow during the expansion and the tear of the intima of the vessels are more likely to induce thrombus formation. Of the 4 cases of restenosis, most of the cases were affected by the below the knee artery. And foreign literature has also been reported, with diabetes accompanied by PAD, the rate of restenosis after PTA is high. [16-17] All of our cases of restenosis are diabetic. Therefore, the continued use of antiplatelet drugs aspirin and clopidogrel is required to prevent or reduce restenosis.

Interventional therapy is minimally invasive, with significant clinical efficacy, safety and fewer complications. It has important clinical significance in the treatment of lower extremity vascular disease.

\section{$5 \quad$ Limitations of This Research}

First of all, the number of cases in this study is small. Second, the time of treatment is short, and the long-term effect of interventional therapy for lower extremity vascular disease needs to be further observed. Third, the study was carried out in a single institution in Macao and failed to fully reflect the actual situation in Macao. Fourth, the number of samples in this study is small, so the bias in data analysis may be caused.

\section{Reference}

1. Sigala F, Menenakos CH, Sigalas P, et al. Transluminal angioplasty of isolated crual aterial lesions in diabetics with critical limb ischemia. Vasa, 2005, 34:186-191.

2. Lonn L, Delle M, Karlstrom L, et al. Should blunt arterial trauma to the extremities be treated with endovascular techniques. J Trauma, 2005, 59:1224-1227.

3. Tsujimura, Takuya, et al. Two-year Clinical Outcomes Post Implantation of EpicTM Self-Expanding Nitinol Stents for the Aortoiliac Occlusive Disease in Patients with Peripheral Arterial Disease. Journal of atherosclerosis and thrombosis (2017): 41152.

4. Global, regional, and national incidence, prevalence, and years lived with disability for 310 diseases and injuries, 1990-2015: a systematic analysis for the Global Burden of Disease Study 2015. Lancet. 388(10053): 1545-1602.

5. Finlayson EV, Birkmeyer JD. Operative mortality with elective surgery in older adults[J]. Eff Clin Pract, 2001, 4:172-177.

6. Baerlocher, Mark Otto, et al. Meta-analysis of drug-eluting balloon angioplasty and drug-eluting stent placement for infrainguinal peripheral arterial disease. Journal of Vascular and Interventional Radiology 26.4 (2015): 459-473.

7. Bosch JL, Hunink MG: Meta-analysis of the results of percutaneous transluminal angioplasty and stent placement for aortoiliac occlusive disease. Radiology, 1997; 204: 87-96.

8. George, Jon C. CRT-300.18 Supera Stent Outcomes In Above-The-Knee Interventions: Effects Of Compression And Elongation (Sake-Compel) Sub-Study. JACC: Cardiovascular Interventions10.3 Supplement (2017): S43.

9. Ichihashi, Shigeo, et al. In Vitro Quantification of Luminal Denudation After Crimping and Balloon Dilatation of Endothelialized Covered Stents. Cardio Vascular and Interventional Radiology (2017): 1-8.

10. de Boer, Sanne W., et al. Short-term Results of the RAPID Ra ndomized Trial of the Legflow P aclitaxel-Eluting Balloon With Supera Stenting vs Supera Stenting Alone for the Treatment of I nterme d iate and Long Superficial Femoral Artery Lesions. Journal of Endovascular Therapy(2017): 1526602817725062.

11. Schneider, Peter A. Evolution and current use of technology for superficial femoral and popliteal artery interventions for claudication. Journal of Vascular Surgery 66.3 (2017): 916-923.

12. Ohki, Takao, et al. Outcomes of the Japanese multicenter Viabahn trial of endovascular stent grafting for superficial femoral artery lesions. Journal of Vascular Surgery(2017).

13. Reijnen, Michel MPJ, et al. 1-Year Results of a Multicenter Randomized Controlled Trial Comparing HeparinBonded Endoluminal to Femoropopliteal Bypass. JACC: Cardiovascular Interventions 10.22 (2017): 2320-2331.

14. Klein, Andrew J., and Charles B. Ross. Endovascular treatment of lower extremity peripheral arterial disease. Trends in cardiovascular medicine 26.6 (2016): 495-512.

15. Fujihara, Masahiko, et al. Angiographic Dissection Patterns and Patency Outcomes After Balloon Angioplasty for Superficial Femoral Artery Disease. Journal of Endovascular Therapy (2017): 1526602817698634. 
16. Faglia E, Clerici G, Losa S, et a1. Limb revascularization feasibility in diabetic patients with critical limb ischemia: Results from a cohoa of 344 consecutive unselected diabetic patients evaluated in 2009[J]. Diabetes Res Clin Pract. 2012, 95(3): 364-371.

17. Faaglia E, Clerici G, Clerissi J, et a1. Long - term prognosis of diabetic patients with critical limb iscbemia: a population—based cohen study[J]. Diabetes Care, 2009, 32(5): 822-827. 